\title{
Genomic evidence that blind cavefishes are not wrecks of ancient life
}

6 Maxime Policarpo ${ }^{1}$, Patrick Laurenti ${ }^{2,7}$, Erik García-Machado ${ }^{3,4}$, Cushla Metcalfe ${ }^{5}$, Sylvie

7 Rétaux ${ }^{*, 6}$ and Didier Casane ${ }^{*, 1,7}$

8

$9 \quad{ }^{1}$ Université Paris-Saclay, CNRS, IRD, UMR Évolution, Génomes, Comportement et

10 Écologie, 91198, Gif-sur-Yvette, France.

112 Université de Paris, Laboratoire Interdisciplinaire des Energies de Demain, Paris, France

$12{ }^{3}$ Department of Biology, Institut de Biologie Intégrative et des Systèmes, Université Laval,

131030 Avenue de la Médecine, Québec City, Québec G1V 0A6, Canada.

$14{ }^{4}$ Centro de Investigaciones Marinas, Universidad de La Habana, Calle 16, No. 114 entre 1ra y 15 3ra, Miramar, Playa, La Habana 11300, Cuba.

$16{ }^{5}$ Independent Researcher, PO Box 21, Nambour QLD 4560, Australia.

$17{ }^{6}$ Université Paris-Saclay, CNRS, Institut des Neurosciences Paris-Saclay, 91190, Gif-sur-

18 Yvette, France.

$19 \quad{ }^{7}$ Université de Paris, UFR Sciences du Vivant, F-75013 Paris, France.

20

21 * Corresponding authors: e-mails: sylvie.retaux@cnrs.fr; didier.casane@egce.cnrs-gif.fr 
25 Cavefishes often have modified eyes, from small but otherwise functional, to highly degenerate structures embedded in a connective tissue and covered by skin. Darwin assumed that these animals are 'wrecks of ancient life', but several genomic studies suggests they are not 'ancient'. The most radical dating shift is for populations of a Mexican cavefish, Astyanax mexicanus, that have been recently estimated to be at the most a few tens of thousands years old. Despite having highly degenerate eyes, the eye-specific genes of A. mexicanus have low levels of decay. Other blind cavefishes we have examined so far are even older, but also can be dated to the Pleistocene. Here, we estimated the age of blindness of two additional fish species by the level of decay of eye-specific genes. Many pseudogenes were identified in the amblyopsid Typhlichthys subterraneus, suggesting that blindness evolved a few million years ago. In contrast, the blind cichlid Lamprologus lethops appears to be a new case of very recent and rapid eye regression, which occurred in deep river water, an environment similar to caves. Genome-wide analyses support the hypothesis that blindness in cavefishes is never very ancient, and ranges from the Early Pliocene to Late Pleistocene. Together with the description of hundreds of cavefish species, our results suggest that surface fishes were able to recurrently and rapidly adapt to caves and similar small dark ecosystems but the resulting highly specialized blind species with a limited distribution may be evolutionary dead-ends in a relatively short time.

Key words: cavefishes, eye regression, eye-specific genes, pseudogenization, molecular dating. 


\section{Introduction}

In On the origin of species, Darwin wrote: "Far from feeling any surprise that some of the cave-animals should be very anomalous, as Agassiz has remarked in regard to the blind fish, the Amblyopsis, and as is the case with the blind Proteus with reference to the reptiles of Europe, I am only surprised that more wrecks of ancient life have not been preserved, owing to the less severe competition to which the inhabitants of these dark abodes will probably have been exposed” (Darwin 1859). Since the middle of the 19th century, thousands of obligate subterranean dwelling animals (troglobionts) have been described, most of them small arthropods (Culver and Pipan 2009; Romero 2009). Among the vertebrates, in addition to the cavefish, Amblyopsis spelaea, and the cave salamander, Proteus anguinus, mentioned by Darwin, many other troglobionts, primarily approximately two hundred and fifty named species of cavefishes (https://cavefishes.org.uk/), and at least 11 salamander species (Romero 2009) have been reported.

In the text quoted above, anomalous animals refers to troglomorphic (cave-related) characters, often present in cave animals, the two most conspicuous being highly degenerate eyes and depigmentation. Caves are very challenging ecosystems and surface animals have to adapt after settling in order to thrive in the long term. Interspecific competition is probably lower because there are fewer predators and other species that are competitors for food resources, but intraspecific competition is high in such a nutrient-limited environment (Poulson and White 1969). The depiction of cavefishes as wrecks of ancient life suggests that regressed traits are the result of relaxed selection, but it does not acknowledge that direct and indirect selection could also be involved in the evolutionary process. For instance, eye degeneration depends on mutations disrupting their development, but both selection and genetic drift could have been implicated in their fixation. In Astyanax mexicanus cavefish, a QTL analysis (Protas, et al. 2007) and indirect arguments based on the energetic cost of eyes (Moran, et al. 2015) suggest that selection for smaller eyes may have been involved in eye degeneration.

Other analyses suggest indirect selection on eye size as the consequence of direct selection on other traits genetically correlated (Yamamoto, et al. 2009; Yoshizawa, et al. 2012; Hinaux, et al. 2016). In addition, if we assume that the first mutations reducing eye size were advantageous in the darkness of caves, other mutations may have accumulated later through genetic drift in small-eyed or blind fish (Retaux and Casane 2013). It has proven very difficult to disentangle these processes because they are not mutually exclusive (Retaux and Casane 2013). In addition, the term "wreck” does not acknowledge that cave animals in general, and 
cavefishes in particular, may evolve constructive traits involved in their adaptation to cave ecosystems (Hinaux, et al. 2016; Soares and Niemiller 2020).

Previous studies have used the level of eye regression to infer the relative age of cavefishes. However, these studies were biased and unreliable because they assumed that eye degeneration is a slow progressive process, lacked an explicit phylogenetic framework, or used phylogenies based on troglomorphic characters (Eigenmann 1909; Poulson and White 1969; Poulson 2001). Our current knowledge of eye development suggests that the idea of a slow, steady and progressive process is misleading because the impact of mutations on eye development can vary enormously, from a minor effect on eye structure and function to the most extreme case in which a single mutation can produce an eyeless phenotype (Loosli, et al. 2003). Today, phylogenetic inference and dating based on gene sequences have superseded those relying on morphological characters. The main advantage of a gene-based phylogenetic approach is that relationship and dating inferences are independent of the analysis of the evolution of morphological traits which are mapped on a priori molecular phylogenies. Assuming that genes used for phylogenetic analyses and troglomorphic traits evolved independently, molecular phylogenies allow us to identify convergent evolution. Moreover, the analysis of genes considered to be under relaxed purifying selection after cave settlement allows us to date the environmental shift from surface to cave and to test hypotheses on the mode and tempo of eye degeneration.

A recent study of the genomes of several cavefish species available at the time allowed us to examine the decay of many eye-specific genes and non-visual opsins, and estimate the age of the shift from surface to cave environment (Policarpo, et al. 2021). This genome-wide analysis demonstrated a low level of gene decay suggesting that these cavefishes originated recently, that is during the Pleistocene (from 2,580,000 to 11,700 years ago). Moreover, the level of eye regression was not correlated with the proportion of eye-specific pseudogenes. However, previous studies based on analyses of one or two genes suggest that other cavefishes could be much more ancient, in particular some among the amblyopsid cavefishes (Dillman, et al. 2011; Niemiller, et al. 2013). Here we sought to further investigate the time of origin of a blind amblyopsid cavefish, Typhlichthys subterraneus, using our genome-wide approach. We also examined the same set of eye-specific genes and non-visual opsins of a blind cichlid, Lamprologus lethops, which inhabits the deep-water of the Congo river. The level of gene decay in Typhlichthys subterraneus supports that blindness may be a few million 
years old in this lineage, indicating that this species may had settled in caves during the

116 Pliocene (from 5.333 million to 2.58 million years ago). In contrast, the very low level of gene decay in Lamprologus lethops indicates that eye regression is very recent, that is it evolved less than 100,000 years ago. These analyses strengthen the hypothesis that once fishes have settled into a dark environment, eyes can degenerate rapidly, reducing in size, sinking into their orbit and becoming covered by skin. In parallel, genes with eye-restricted expression accumulate LoF mutations, but this process is comparatively much slower.

Results

In a previous study (Policarpo, et al. 2021), we defined three gene sets based on the zebrafish genome: 95 vision genes, 42 circadian clock genes and 257 pigmentation genes (fig. 1). In the present analysis, we looked for likely LoF mutations (i.e. premature STOP codons, losses of START and STOP codons, losses of intron splice sites and small indels leading to frameshifts) in homologs from the genomes of a blind cavefish from North America, the Southern Cavefish Typhlichthys subterraneus (Amblyopsidae) and a blind fish living in deep water of the Congo River in Central Africa, Lamprologus lethops (Cichlidae). For each surface fish for which a genome was available, Percopsis transmontana and Lamprologus tigripictilis, respectively, and a more distantly related surface species, Gadus morhua and Neolamprologus pulcher, respectively (fig. 2). mutations (47) were found in vision genes, while only 2 LoF were found in circadian clock genes and 6 in pigmentation genes. Most LoF mutations in vision genes (43/47) were found in T. subterraneus (supplementary fig. S1 and fig. S2, Supplementary Material online).

144 In order to test if these LoF mutations were distributed randomly within the genes, that is, 145 they were not clustered at the 3' end of the genes where their deleterious effect would be 146 smaller, we computed the effective number of gene segments generated by LoF mutations and compared this value with simulations of random distributions of mutations within genes 
148 (Policarpo, et al. 2021). We found that premature STOP codons and frameshifts are

149 distributed randomly throughout the coding sequences (supplementary fig. S3,

150 Supplementary Material online).

151

152 We counted the number of pseudogenes in all six species, that is, the number of genes

153 carrying at least one LoF mutation (fig. 1 and fig. 2). Among the vision genes, we identified

15427 pseudogenes in T. subterraneus, but only 3 pseudogenes in L. lethops. The LoF mutations

155 found in L. lethops (fig. 1, supplementary fig. S2, Supplementary Material online) have

156 been reported in a previous study (Aardema, et al. 2020). A gene, opn3, was also reported as

157 pseudogenized in this publication, but it was considered a functional gene in our study.

158 Indeed, the frameshift reported in opn3 is probably an artefact as this gene is not where the

159 deletion, at the origin of the frameshift, was found. We did not find any LoF mutations in this

160 gene (for more details, see supplementary fig. S4, Supplementary Material online).

161 The percentage of vision pseudogenes in T. subterraneus (44\%) is the highest ever reported in

162 cavefishes. In contrast, the percentage of vision pseudogenes is very low in L. lethops (4\%).

163 No circadian clock pseudogene was found in L. lethops and only one pseudogene (per2) in $T$.

164 subterraneus. The loss of per2 is recurrent in cavefishes, as pseudogenes of per2 have been

165 identified in S. rhinocerous and P. andruzzii (Yang, et al. 2016; Ceinos, et al. 2018; Policarpo,

166 et al. 2021). A circadian clock pseudogene (cry-dash) was found in the surface fish $L$.

167 tigripictilis, however, the specimen was heterozygous for this gene. Two pigmentation

168 pseudogenes (trpm $1 b$ and mreg) were identified in T. subterraneus and one pseudogene

169 (oca2) in L. lethops. This mutation has already been reported in a previous study (Aardema, et

170 al. 2020). In the surface fish L. tigripictilis, a pseudogene (pts) was found, however, the

171 specimen was also heterozygous for the gene (fig. 2, Table S1, Supplementary Material

172 online).

173

174 Together, findings from the present study and a previous study (Policarpo, et al. 2021) has

175 allowed us to estimate that among the set of 95 vision genes, 46 have been found

176 pseudogenized in at least one cave species, that is, $48 \%$ of the gene set. In sharp contrast, only

1774 pseudogenes were identified among 42 circadian clock genes, and 18 pseudogenes among

178 the 257 pigmentation genes, that is, $10 \%$ and $7 \%$ respectively.

179 
In T. subterraneus, among 62 vision genes, $43 \mathrm{LoF}$ mutations were found in 27 pseudogenes. More precisely, there were 17 pseudogenes with 1 mutation, 7 with 2 mutations, 1 with 3 mutations, 1 with 4 mutations and 1 with 5 mutations. This distribution was compared with expected distributions obtained for different numbers of neutral genes ranging from 27 (44\%) to $62(100 \%)$. A better fit between the observed and expected distribution was found when at least $80 \%$ of genes were assumed to be neutral sequences (fig. 3 ). This result is congruent with the estimation based on the distribution of LoF mutations per pseudogene in Lucifuga dentata (Policarpo, et al. 2021) (fig. 3). Moreover, the higher percentage of pseudogenes with more than one LoF mutation in T subterraneus than in $L$. dentata is congruent with the higher percentage of pseudogenes (44\% vs 25\%) indicating that $T$. subterraneus is the oldest cavefish species.

Another approach to tackle this issue involved the analysis of the number of vision genes pseudogenized in both species. There are 27 pseudogenes among 62 vision genes in $T$. subterraneus (present study) and 19 pseudogenes among 76 vision genes in L. dentata (Policarpo, et al. 2021). Moreover, among 58 vision genes identified in both species, there are 10 genes - opn7b, parapinopsin-1, parietopsin, cryaa, crybb1, crybgx, crygn2, grk1b, grk7a and $g c 2$ - pseudogenized in both species. As expected for two distantly related blind species, no shared LoF mutation was found in orthologous pseudogenes (supplementary Table S2, Supplementary Material online). Assuming that all vision genes are dispensable and have the same LoF mutation rate, we can compute the expected number of genes pseudogenized in parallel in two species among a set of vision genes studied in both species (probability of pseudogenization in one species $x$ probability of pseudogenization in the other species $x$ number of genes), that is $27 / 62 \times 19 / 76 \times 58=6.3$. A script was written to compute the distribution of the probabilities of the number of shared pseudogenes, in a range between 0 and 14, taking into account that 14 pseudogenes were found in L. dentata and 25 in $T$. subterraneus among the 58 vision genes found in both species (supplementary fig. S5,

210 Supplementary Material online). The expected value (6.3) and the low probability (0.01) of

211 finding 10 shared pseudogenes suggest a significant excess of shared pseudogenes that could 
213 realistic model taking into account the length and the number of introns of each gene to

214 estimate the relative LoF mutation rate of each gene, a probability higher than $5 \%$ was found

215 for a number of shared pseudogenes in a range between 5 and 10 (supplementary fig. S5,

216 Supplementary Material online). This result is another evidence that most, if not all, vision

217 genes are dispensable in these blind species.

To date the relaxation of purifying selection on vision genes in T. subterranues, L. lethops and

A. mexicanus, for each species, we used the number of pseudogenes among vision genes and the LoF mutation rate per generation estimated as described in a previous publication (Policarpo, et al. 2021). The highest probability of finding 27 pseudogenes among 62 genes in T. subterraneus was obtained for relaxed selection starting 569,570 generations ago (probability > 5\% between 447,390 and 712,830 generations). For L. lethops (3 pseudogenes among 79 genes), the time since relaxed selection was estimated to 28,970 generations ago (probability > 5\% between 8,670 and 68,620 generations). For A. mexicanus (1 pseudogene among 85 genes), the time since relaxed selection was estimated to 8,560 generations ago (probability > 5\% between 450 and 38,580 generations). For L. dentata (19 pseudogenes among 76 genes), the estimate was obtained in a previous study (Policarpo, et al. 2021), 367,780 generations ago (probability $>5 \%$ in a range between 273,990 and 480,980 generations)(fig. 4).

For each species, we also computed this probability using only a subset of vision genes found pseudogenized in at least one among the other cavefishes, increasing the likelihood that the genes examined are dispensable in caves. Excluding T. subterraneus, 33 genes were found with at least one LoF mutation in the other cave species. Twenty-one of these genes were identified in T. subterraneus of which 13 were pseudogenes. The highest probability of finding 13 pseudogenes among 21 genes was obtained for relaxed selection starting 910,940 generations ago (probability $>5 \%$ between 557,730 and 1,401,750 generations). Excluding $L$. lethops, 46 genes with a LoF were found in the other cave species. Forty-one of these were identified in L. lethops, of which 2 were pseudogenes. The time since relaxed selection was estimated to be 35,200 generations ago (probability > 5\% between 6,640 and 103,250 generations). Excluding A. mexicanus, 47 genes with a LoF were found in the other cave species. Forty-four of these were identified in A. mexicanus, of which one was a pseudogene. 
5\% between 790 and 67,740 generations). Excluding L. dentata, 41 genes with a LoF were found in the other cave species. Thirty-five of these were identified in L. dentata, of which 13 were pseudogenes. The time since relaxed selection was estimated to be 559,690 generations ago (probability $>5 \%$ between 365,220 and 814,710 generations).

\section{Discussion}

We discuss the patterns of gene decay among different gene sets in various cavefishes. Then, we show how our analyses of the genomes of a blind cavefish and a deep-water blind fish strengthen our hypotheses on the pace of eye loss and the evolutionary fate of cave-adapted

\section{8 fishes.}

\section{Patterns of gene decay}

In a previous study, we defined a set of genes we called vision genes that are most likely to be dispensable in a dark environment and can carry LoF mutations without a large impact on overall fitness. They are primarily eye-specific genes involved in visual phototransduction, but we also included a set of non-visual opsin that showed similar gene decay (Policarpo, et al. 2021). In this publication, our analysis of gene decay in $L$. dentata suggested that most, if not all, vision genes are dispensable in blind cave fishes, however only $25 \%$ of L. dentata vision genes have LoF mutations. In the present study, we found that $44 \%$ of the vision genes of $T$. subterraneus have LoF mutations, an observation strongly strengthening our hypothesis. In $T$. subterraneus, the distribution of LoF mutations per genes is similar to the one in $L$. dentata and in accordance with the assumption that most vision genes are under relaxed purifying selection in the darkness of caves.

273 The analysis of seven cavefishes identified pseudogenes in $48 \%$ of the 95 vision genes. We

274 have shown that this set of genes is a useful tool for estimating the time of origin of blindness

275 and the extent of common origin and parallel evolution among closely related cavefishes.

276 However, only the analysis of other cavefish genomes may allow us to unambiguously

277 identify the subset of vision genes that are dispensable in the dark. 
281 Assuming that eye regression is a progressive process and that cavefishes can be ordered on a ladder of degeneration, eye regression has been used to estimate the age of different cavefish lineages (Wilkens, et al. 1989). However, a well-established and independent phylogenetic framework and independent evidence of the age of eye regression are necessary to understand the dynamic of eye regression and the diversity of its modality in different cavefish lineages. The very low decay in vision genes in Astyanax mexicanus cavefish, despite their highly degenerate eyes, indicates that $A$. mexicanus has lost eyesight very recently (Fumey, et al. 2018; Policarpo, et al. 2021). The very similar pattern of gene decay observed in however, while the eyes are very small and sunk under the skin, their general organization is much better preserved. On the other hand, both cavefishes that have accumulated many vision pseudogenes, that is Lucifuga dentata and Typhlichthys subterraneus, have highly degenerate eyes. There are probably many evolutionary pathways leading to eye regression in cavefishes, some through intermediary stages involving first eye size reduction but normal development, while in other cases through changes in early development leading to highly degenerate eyes. Both chance and environmental constraints may be involved in this process. In particular, it is possible that some blind fishes need to retain the capacity to detect sources of light.

\section{Conclusion}

The analysis of two recently published genomes of blind fishes, one living in caves and the other in the deep-water of a river, strengthens our hypothesis that blind cavefishes are not very ancient and most of them lost eye function during the Pliocene and Pleistocene. The recent eye regression in $L$. lethops indicates that eyes can sink rapidly without other important morphological changes, or as in A. mexicanus, with extensive eye degeneration. In both cases, the number of eye-specific pseudogenes is very small, two and one respectively. In other words, eyes can sink rapidly but eye-specific genes rust slowly in cavefishes. Among blind cavefishes for which the genome is available, several lines of evidence suggest that the oldest may be the amblyopsid T. subterraneus, in which $44 \%$ of vision genes are pseudogenized. Nevertheless, other North American amblyopsids may be older (Niemiller, et

311 al. 2013). When the genomes of these species will be available, the analysis of the vision genes should clarify the age of these cavefishes and also to which extent cave amblyopsids evolved independently. 
314 The analysis of the genome of $T$. subterraneus allowed us to identify a blind cavefish that is

315 probably older than L. dentata, which was previously considered the oldest cavefish among 316 those with sequenced genomes, but this does not refute our current working hypothesis that

317 blind cavefishes cannot thrive more than a few millions years in cave ecosystems.

\section{Materials and Methods}

Vision, circadian clock and pigmentation gene sets

The three sets of genes, vision, circadian clock and pigmentation, have been previously studies (Li, et al. 2013; Lorin, et al. 2018). The complete list of genes with standard identifiers (https://zfin.org/) is given in fig. 1.

\section{Construction of data sets}

Danio rerio sequences were used as queries for Blast and tBlastn 2.6.0+ (Altschul, et al. 1990) to the genome of Percopsis transmontana (GCA_900302285.1), Gadus morhua (gadMor1), Neolamprologus pulcher (GCF_000239395.1) and Typhlichthys subterraneus SAMtools v1.9 faidx ( $\mathrm{Li}$ 2011). Coding sequences and introns were predicted using EXONERATE v2.4.0 (Slater and Birney 2005) (model protein2genome, using Danio rerio protein sequences as query).

340 BAM files of Lamprologus lethops and Lamprologus tigripictilis reads aligned to the genome of Neolamprologus pulcher were downloaded from NCBI (SRR10811633, SRR10811634). SAMtools BCFtools v1.9 (Li 2011) was used to generate a consensus genome (BCFtools consensus) from which genes were predicted following the method described above. Coding sequences were classified as "complete" if the complete CDS was retrieved or "incomplete" otherwise. If less than $10 \%$ of a coding sequence was found, the gene was classified as "Not Found” and it was not further analyzed. A CDS was classified as a "pseudogene” if at least 
one LoF mutation was found (premature STOP codon, loss of the initiation codon, loss of the STOP codon, indel leading to a frameshift, mutations at intron splice sites).

\section{Phylogenetic analyses}

Orthologous and paralogous relationships between genes were inferred through phylogenetic analyses. Coding sequences were trimmed and aligned with MACSE v2.03 program trimNonHomologousFragments and alignSequences (Ranwez, et al. 2018). Frameshifts and internal stop codons were then removed with the MACSE v2.03 progam exportAlignment. For each alignment, DNA sequences were translated into protein sequences and a maximum likelihood phylogenetic tree was inferred using IQ-TREE v1.6.12 (Nguyen, et al. 2015) with the optimal model found by ModelFinder (Kalyaanamoorthy, et al. 2017). Robustness of the nodes was evaluated with 1,000 ultrafast bootstraps (Hoang, et al. 2018). Trees were rooted and visualized using iTOL v6 (Letunic and Bork 2007).

\section{Estimation of the proportion of neutral vision genes in cavefishes}

The distribution of LoF mutations per gene in T. subterraneus was used to estimate the proportion of neutral genes using the method described previously in (Policarpo, et al. 2021). In brief, 10,000 simulations of the distribution of 43 mutations in a random sample of $\mathrm{V}$ genes from the $62 \mathrm{~T}$. subterraneus vision genes was generated. $\mathrm{V}$ ranged from the number of observed pseudogenes (27), which is the minimal number of neutral genes, to the total number of genes (62). The length of the coding sequence and the number of introns of each gene were taken into account to compute the relative mutation rate.

Then, we computed the expected number of vision genes pseudogenized in both $T$.

subterraneus and L. dentata, assuming that all vision genes are dispensable and have the same LoF mutation rate. The following formula was used: $\mathrm{E}=\mathrm{P}_{\mathrm{Ts}} \mathrm{X} \mathrm{P}_{\mathrm{Ld}} \mathrm{X} \mathrm{N}$, where $\mathrm{P}_{\mathrm{Ts}}$ is the probability that a vision gene is pseudogenized in T. subterraneus, $\mathrm{P}_{\mathrm{Ld}}$ is the probability that a vision gene is pseudogenized in L. dentata, and $\mathrm{N}$ is the number of vision genes found in both species.

Given that of the 58 vision genes found in both species, 14 genes were pseudogenized in $L$. dentata, 25 in T. subterraneus, and 10 were shared, a custom R script was used to evaluate if this number of shared pseudogenes was likely under the hypothesis that all vision genes are 
380

381

382

383

384

385

386

387

388

389

390

391

392

393

394

395

396

397

398

399

400

401

402

403

404

405

406

407

408

409

410

411

412

dispensable. The script generated 10,000 simulations of random pseudogenization of the genes, taking into account the length and the number of introns of each gene to compute its relative mutation rate. For each simulation, the number of shared pseudogenes was recorded, allowing us to estimate the probability of obtaining a given number of shared pseudogenes in the range between 0 and 14 (supplementary fig. S5, Supplementary Material online).

\section{Dating relaxation of purifying selection on vision genes in L. lethops and $T$.} subterraneus from pseudogene numbers

We used the method previously described (Policarpo, et al. 2021). Briefly, we computed the probability than $\mathrm{D}$ genes among $\mathrm{T}$ genes accumulated a LoF mutation taking into account the mean gene length. This method requires an estimate of the LoF mutation rate per generation. Assuming a transition/transversion ratio in vision genes of 1.68 computed with PAML v4.9h (Yang 2007) and taking into account the codon usage in vision genes, we found that the probability that a single mutation led to a premature stop codon was 0.031 . In $T$. subterraneus, 12 premature stop codons and 27 frameshifts were found in vision genes. Thus, the probability that a mutation is a frameshift was $(27 / 12) \times 0.031=0.069$.

The probability of splice site mutation was $4 \mathrm{x}$ (total intron number / total cds length) $=4 \mathrm{x}$ $(255 / 48703)=0.021$. The probability of start loss was equal to $3 \mathrm{x}$ (number of genes / total cds length $)=3 \times(62 / 48703)=0.0038$ and the probability of stop loss was equal to $0.852 *$ (Start loss probability) $=0.0033$. Thus $\mu_{\text {LoF }}$ was equal to $0.128 \mu$ with $\mu$ set to $10^{-8}$. As a very low number of pseudogenes were found in L. lethops, the LoF mutation rate found in T. subterraneus was also used for dating relaxation of selection in L. lethops and A. mexicanus. Dating of $L$. dentata was estimated using the LoF mutation rate $(0.0717 \mu)$ reported in a previous study (Policarpo, et al. 2021).

\section{$\mathrm{R}$ analyses}

R script files for reproducing analyses and figures is available in supplementary files (see Data Availability) and on GitHub (https://github.com/MaximePolicarpo/Genomic-evidencethat-cavefishes-are-not-wrecks-of-ancient-life). A list of R packages used is given below, with a reference to a publication if available or an URL otherwise: plotrix v3.7-7 (Lemon 2006), data.table v1.12.8 (https://cran.r-project.org/web/packages/data.table/index.html), dplyr v0.8.5 (https://cran.r-project.org/web/packages/dplyr/index.html), tidyverse v1.3.0 (Wickham, et al. 
413 2019), purr v0.3.3 https://cran.r-project.org/web/packages/purrr/index.html, ggplot2 v3.3.0

414 (Wickham 2016), ggpubr v0.2.5 (https://cran.r-project.org/web/packages/ggpubr/index.html), gtools v3.8.2 (https://cran.r-project.org/web/packages/gtools/index.html), forcats v0.5.0

416 (https://cran.r-project.org/web/packages/forcats/index.html), FSA v0.8.30 (Ogle, et al. 2020), 417 patchwork v1.0.0 (https://cran.r-project.org/web/packages/patchwork/index.html), quantable 418 0.3.6 (https://cran.r-project.org/web/packages/quantable/index.html), reticulate v1.15 419 (https://cran.r-project.org/web/packages/reticulate/index.html), sjPlot v2.8.3 (Lüdecke 2020), 420 gridExtra v2.3 (https://cran.r-project.org/web/packages/gridExtra/index.html), ape v5.3

421 (Paradis and Schliep 2019) and phytools v0.7-47 (Revell 2012).

422

\section{Data Availability}

424

425 All data and results of the analyses performed in this study are available for download at 426 figshare (https://figshare.com/articles/dataset/Supplementary_files/14237279): three tables 427 with all gene sequences and their genomic positions (one table per gene data set); three tables 428 with a description of LoF mutations (one table per gene data set); Exonerate raw results; Gene 429 alignments, IQ-TREE results and phylogenetic trees in PDF; a R script to reproduce the 430 analyses.

431

432 Supplementary Material

433

434 Supplementary data are available at Genome Biology and Evolution.

435

\section{Acknowledgments}

437

438 This work was supported by a collaborative grant from Agence Nationale de la Recherche

439 (BLINDTEST to S.R. and D.C.) and from Institut Diversité Ecologie et Evolution du Vivant 440 (to S.R. and D.C). 
442

443

444

445

446

447

448

449

450

451

452

453

454

455

456

457

458

459

460

461

462

463

464

465

466

467

468

469

470

471

472

473

474

\section{Figure Legends}

Fig 1: Gene sets and pseudogenes. (A) Vision genes. (B) Circadian clock genes. (C) Pigmentation genes. Pseudogenes are coloured according the species in which they were found. In candidate gene studies, only few genes were examined, whereas most genes were inspected in our genome-wide analyses. Gene copy number variations: there are four copies of $R h 2$ in zebrafish whereas there is only one $R h 2$ gene in $T$. subterraneus; there is one opn6a gene in zebrafish whereas there are two opn6a genes in T. subterraneus. Data for previously studied cavefishes (A. mexicanus, Lucifuga spp. and Sinocyclocheilus spp.) have been redrawn from Policarpo et al. (2021), for comparison.

Fig 2: Phylogeny and pseudogene mapping. For each gene set, the number of pseudogenes found and the number of gene examined in a species are given to the right of the species name. het: heterozygous. Data for previously studied cavefishes (A. mexicanus, Lucifuga spp. and Sinocyclocheilus spp.) have been redrawn from Policarpo et al. (2021), for comparison.

Fig 3: Estimation of the number of neutral genes in T. subterraneus. Red lines: observed distribution. Expected distributions were obtained for different numbers of neutral genes among 62 genes: between 27 and 62 (each expected distribution was obtained with 10,000 simulations). The distributions for L. dentata have been redrawn from Policarpo et al. (2021), for comparison.

Fig 4: Dating of the relaxation of purifying selection on vision genes. Solid lines: probabilities of finding 27 pseudogenes among 62 vision genes in T. subterraneus, 19 pseudogenes among 76 genes in L. dentata, three pseudogenes among 79 vision genes in $L$. lethops and one pseudogene among 85 vision genes in A. mexicanus, according to the time in caves (generations) and assuming relaxed selection on the whole vision gene set in this environment. Dashed lines: probabilities of finding pseudogenes using only genes found pseudogenized in other cave species, increasing the likelihood that the genes examined are dispensable in caves. The number of generations for which the highest probability was found is reported. 
Aardema ML, Stiassny MLJ, Alter SE. 2020. Genomic Analysis of the Only Blind Cichlid Reveals Extensive Inactivation in Eye and Pigment Formation Genes. Genome Biol Evol 12:1392-1406. Altschul SF, Gish W, Miller W, Myers EW, Lipman DJ. 1990. Basic local alignment search tool. J Mol Biol 215:403-410.

Ceinos RM, Frigato E, Pagano C, Fröhlich N, Negrini P, Cavallari N, Vallone D, Fuselli S, Bertolucci C, Foulkes NS. 2018. Mutations in blind cavefish target the light-regulated circadian clock gene, period 2. Scientific Reports 8:8754.

Culver DC, Pipan T. 2009. The Biology of Caves and Other Subterranean Habitats. Oxford: Oxford University Press.

Darwin CR. 1859. On the origin of species by means of natural selection, or the preservation of favoured races in the struggle for life. London: John Murray.

Dillman CB, Bergstrom DE, Noltie DB, Holtsford TP, Mayden RL. 2011. Regressive progression, progressive regression or neither? Phylogeny and evolution of the Percopsiformes (Teleostei, Paracanthopterygii). Zoologica Scripta 40:45-60.

Eigenmann CH. 1909. Cave vertebrates of America : a study in degenerative evolution. Washington, D.C.: Carnegie Institution of Washington.

Fumey J, Hinaux H, Noirot C, Thermes C, Rétaux S, Casane D. 2018. Evidence for late Pleistocene origin of Astyanax mexicanus cavefish. Bmc Evolutionary Biology 18:43. Hinaux H, Devos L, Blin M, Elipot Y, Bibliowicz J, Alié A, Rétaux S. 2016. Sensory evolution in blind cavefish is driven by early embryonic events during gastrulation and neurulation. Development 143:4521.

Hoang DT, Chernomor O, von Haeseler A, Minh BQ, Vinh LS. 2018. UFBoot2: Improving the Ultrafast Bootstrap Approximation. Molecular Biology and Evolution 35:518-522.

Kalyaanamoorthy S, Minh BQ, Wong TKF, von Haeseler A, Jermiin LS. 2017. ModelFinder: fast model selection for accurate phylogenetic estimates. Nature Methods 14:587-589. Lemon J. 2006. Plotrix: A Package in the Red Light District of R. R-News 6. Letunic I, Bork P. 2007. Interactive Tree Of Life (iTOL): an online tool for phylogenetic tree display and annotation. Bioinformatics 23:127-128.

Li H. 2011. A statistical framework for SNP calling, mutation discovery, association mapping and population genetical parameter estimation from sequencing data. Bioinformatics 27:29872993.

Li Y, Li G, Wang H, Du J, Yan J. 2013. Analysis of a Gene Regulatory Cascade Mediating Circadian Rhythm in Zebrafish. Plos Computational Biology 9:e1002940. Loosli F, Staub W, Finger-Baier KC, Ober EA, Verkade H, Wittbrodt J, Baier H. 2003. Loss of eyes in zebrafish caused by mutation of chokh/rx3. EMBO reports 4:894-899. Lorin T, Brunet FG, Laudet V, Volff J-N. 2018. Teleost Fish-Specific Preferential Retention of Pigmentation Gene-Containing Families After Whole Genome Duplications in Vertebrates. G3: Genes|Genomes|Genetics 8:1795-1806.

Lüdecke D. 2020. sjPlot: Data Visualization for Statistics in Social Science. R package version 2.8.5.

Moran D, Softley R, Warrant EJ. 2015. The energetic cost of vision and the evolution of eyeless Mexican cavefish. Science Advances 1:e1500363.

Nguyen L-T, Schmidt HA, von Haeseler A, Minh BQ. 2015. IQ-TREE: A fast and effective Evolution 32:268-274. 
Niemiller ML, Fitzpatrick BM, Shah P, Schmitz L, Near TJ. 2013. Evidence for repeated loss of selective constraint in rhodopsin of amblyopsid cavefishes (teleostei: amblyopsidae). Evolution 67:732-748. Ogle DH, Wheeler P, Dinno A. 2020. FSA: Fisheries Stock Analysis. R package version 0.8.30.

Paradis E, Schliep K. 2019. ape 5.0: an environment for modern phylogenetics and evolutionary analyses in R. Bioinformatics 35:526-528.

Policarpo M, Fumey J, Lafargeas P, Naquin D, Thermes C, Naville M, Dechaud C, Volff J-N, Cabau C, Klopp C, et al. 2021. Contrasting gene decay in subterranean vertebrates: insights from cavefishes and fossorial mammals. Molecular Biology and Evolution 38:589-605. Poulson TL. 2001. Morphological and physiological correlates of evolutionary reduction of metabolic rate among amblyopsid cave fishes. In: Romero A, editor. The biology of hypogean fishes. Dordrecht: Springer Netherlands. p. 239-249.

Poulson TL, White WB. 1969. The Cave Environment. Science 165:971-981.

538

539

540

541

542

543

544

545

546

547

548

549

550

551

552

553

554

555

556

557

558

559

560

561

562

563

564

565

566 Protas M, Conrad M, Gross JB, Tabin C, Borowsky R. 2007. Regressive evolution in the Mexican cave tetra, Astyanax mexicanus. Current Biology 17:452-454.

Ranwez V, Douzery EJP, Cambon C, Chantret N, Delsuc F. 2018. MACSE v2: Toolkit for the Alignment of Coding Sequences Accounting for Frameshifts and Stop Codons. Molecular Biology and Evolution 35:2582-2584.

Retaux S, Casane D. 2013. Evolution of eye development in the darkness of caves: adaptation, drift, or both? Evodevo 4:26.

Revell LJ. 2012. phytools: an R package for phylogenetic comparative biology (and other things). Methods in Ecology and Evolution 3:217-223.

Romero A. 2009. Cave Biology: Life in Darkness. Cambridge: Cambridge University Press. Slater GSC, Birney E. 2005. Automated generation of heuristics for biological sequence comparison. BMC Bioinformatics 6:31.

Soares D, Niemiller ML. 2020. Extreme Adaptation in Caves. The Anatomical Record 303:15-23.

Wickham H. 2016. ggplot2: Elegant Graphics for Data Analysis. New York: Springer-Verlag Wickham H, Averick M, Bryan J, Chang W, McGowan L, François R, Grolemund G, Hayes A, Henry L, Hester J, et al. 2019. Welcome to the Tidyverse. Journal of Open Source Software 4:1686.

Wilkens H, Strecker U, Yager J. 1989. Eye reduction and phylogenetic age in ophidiiform cave fish. Zeitschrift Fur Zoologische Systematik Und Evolutionsforschung 27:126-134. Yamamoto Y, Byerly MS, Jackman WR, Jeffery WR. 2009. Pleiotropic functions of embryonic sonic hedgehog expression link jaw and taste bud amplification with eye loss during cavefish evolution. Developmental Biology 330:200-211.

Yang J, Chen X, Bai J, Fang D, Qiu Y, Jiang W, Yuan H, Bian C, Lu J, He S, et al. 2016. The Sinocyclocheilus cavefish genome provides insights into cave adaptation. BMC Biol 14:1-13. Yang Z. 2007. PAML 4: Phylogenetic Analysis by Maximum Likelihood. Molecular Biology and Evolution 24:1586-1591.

Yoshizawa M, Yamamoto Y, O'Quin KE, Jeffery WR. 2012. Evolution of an adaptive behavior and its sensory receptors promotes eye regression in blind cavefish. BMC Biol 10:108. 
Genome-wide studies

\section{Pseudogene in:}

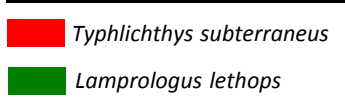

Astyanax mexicanus Pachón cavefish

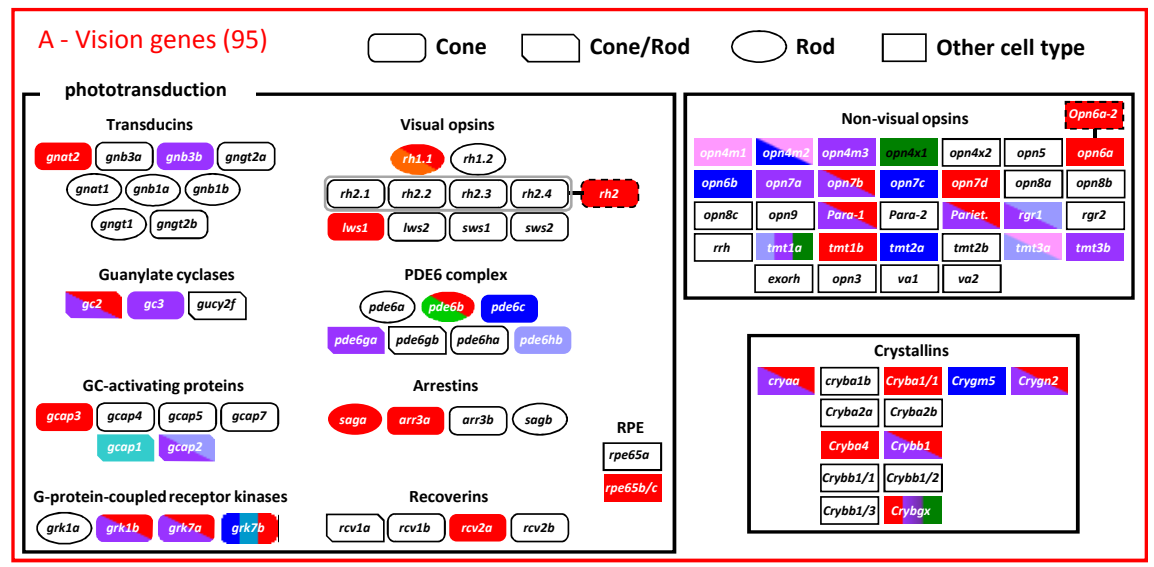

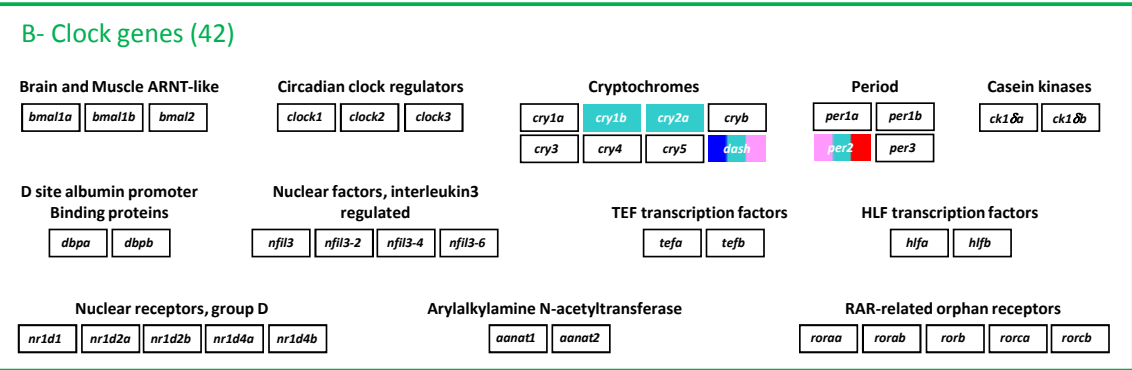

\section{Candidate gene studies}

Amblyopsidae cavefishes

Phreatichthys andruzzii

Oreonectes daqikongensis

C - Pigmentation genes (257)

Melanophore, iridophore, xantophore and leucophore development

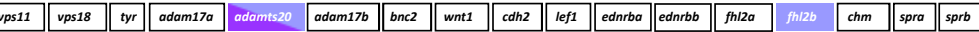

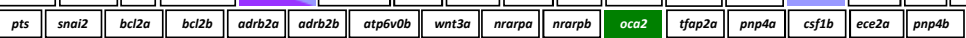

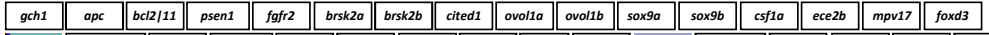

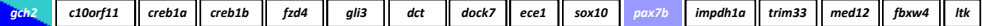

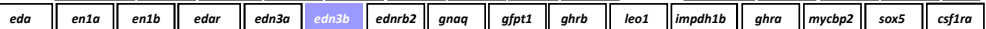

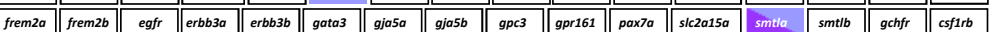

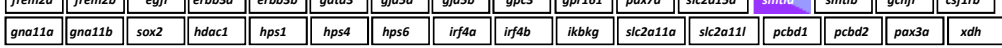

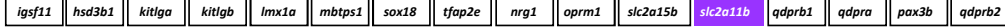

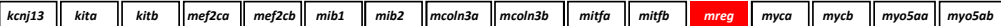

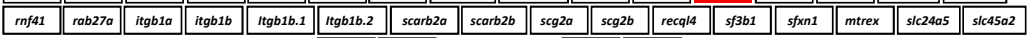

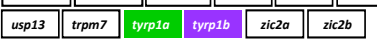

Melanosome transport \begin{tabular}{l|l|l|l|l|l|l|l|l|l}
\hline ippk & dctn2 & tmem 33 & myo7ad & map2k1 \\
\hline
\end{tabular}

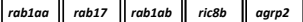

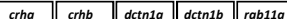
\begin{tabular}{|l|l|l|l|l|l|}
\hline crha & crhb & dctn1a & dctn1b & rabi1a \\
\hline \hline tpcn2 & mlpha & rabsip & mlphb & rabsa \\
\hline
\end{tabular}

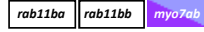

Melanosome biogenesis and components

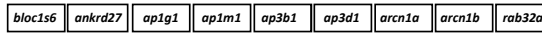

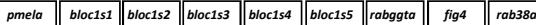

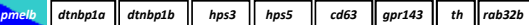

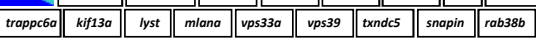

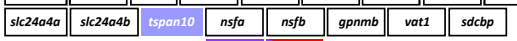
trpm1a trpm $16^{-10}$

Melanogenesis regulation

\begin{tabular}{|l||l|l|l||l|l||l|}
\hline asip1 & atrm & myg1 & cknn & corin & ctns & gart \\
\hline
\end{tabular}

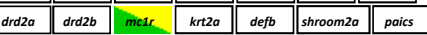
\begin{tabular}{|l|l||l||l|l|l|l|}
\hline slc7a11 & ugt1as & nf1a & nf1b & ostm1 & shroom2b \\
\hline
\end{tabular} \begin{tabular}{|l|l|l|l|l|l|}
\hline \hline mgrn1a & pah & pomca & pomcb & zeb2a & zeb2b \\
\hline
\end{tabular} 
(2) eyes

- small eyes

$\star$ whole genome duplication

( ) no eyes

1 shared pseudogene

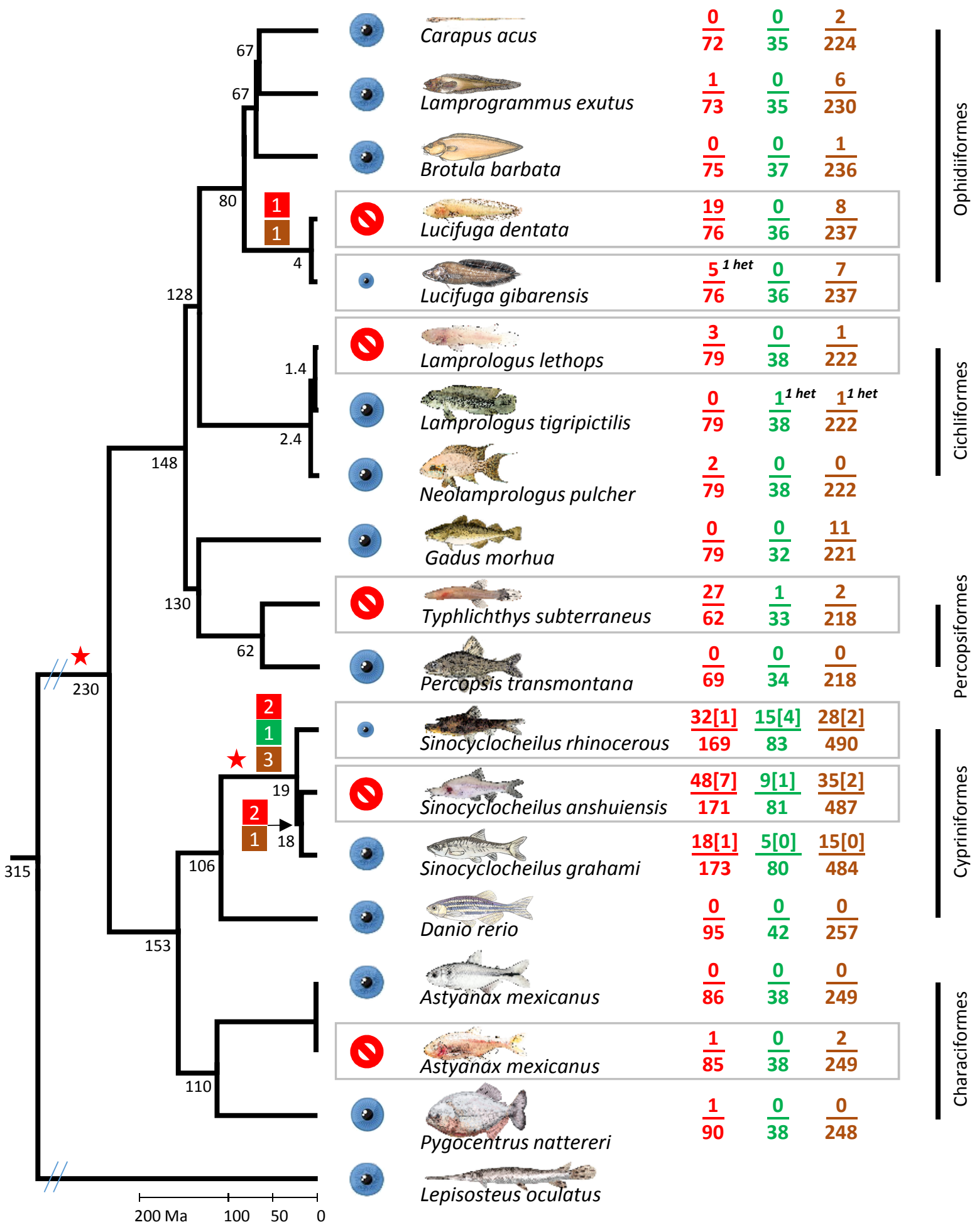




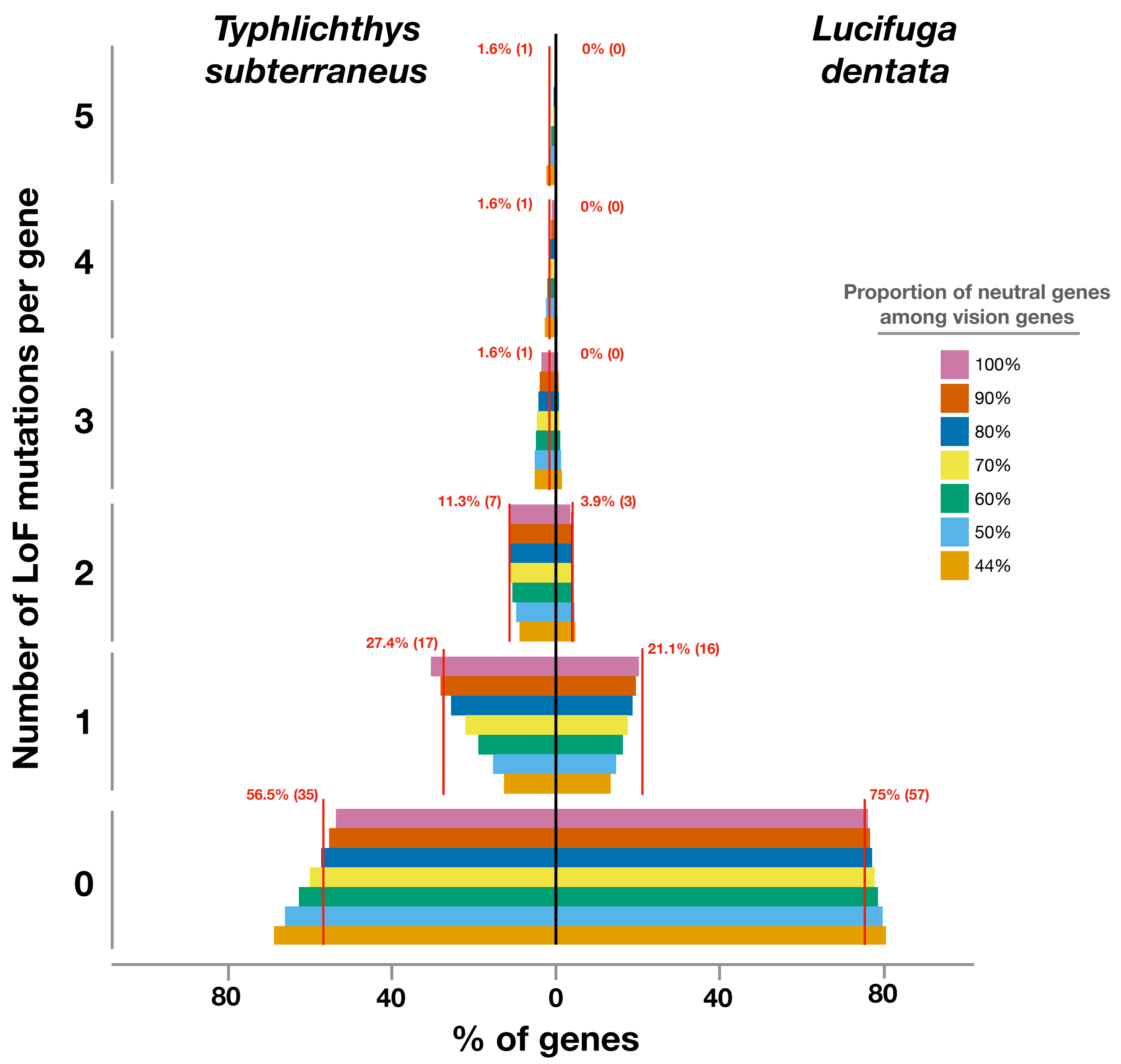




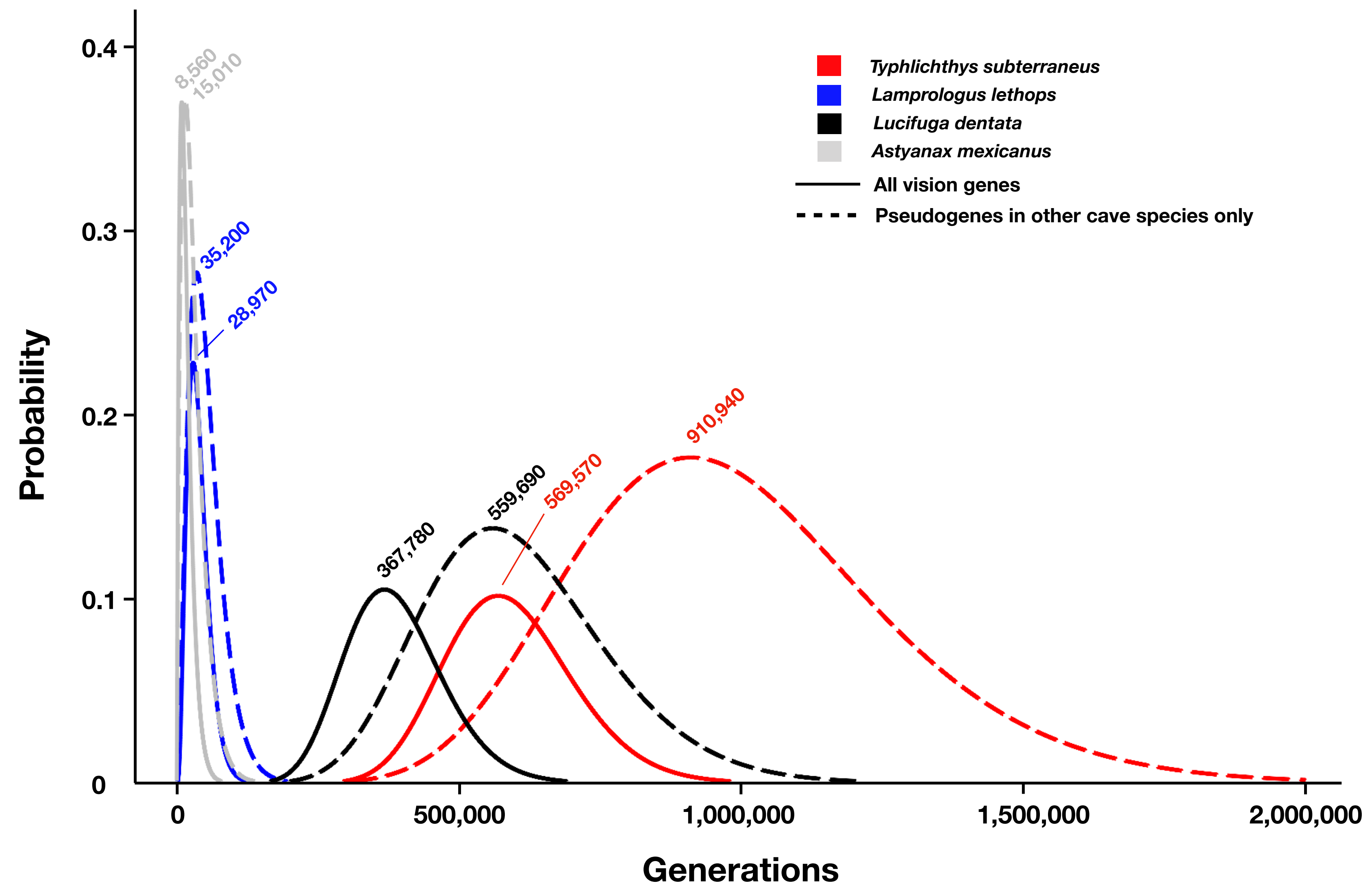

